\title{
Surgical implications of recent modalities for parathyroid imaging
}

\author{
Beata Wojtczak ${ }^{1}$, Joanna Syrycka ${ }^{2}$, Krzysztof Kaliszewski ${ }^{1}$, Jerzy Rudnicki ${ }^{1}$, Marek Bolanowski ${ }^{2}$, \\ Marcin Barczyński ${ }^{3}$
}

${ }^{1}$ Department of General, Minimally Invasive and Endocrine Surgery, ${ }^{2}$ Department of Endocrinology, Diabetes and Isotope Therapy, Wroclaw Medical University, Wroclaw, Poland; ${ }^{3}$ Department of Endocrine Surgery, Third Chair of General Surgery, Jagiellonian University Medical College, Krakow, Poland

Contributions: (I) Conception and design: B Wojtczak; (II) Administrative support: All authors; (III) Provision of study materials or patients: All authors; (IV) Collection and assembly of data: All authors; (V) Data analysis and interpretation: All authors; (VI) Manuscript writing: All authors; (VII) Final approval of manuscript: All authors.

Correspondence to: Beata Wojtczak, MD, PhD. Department of General, Minimally Invasive and Endocrine Surgery, Wroclaw Medical University, Borowska Street 213, 50-556, Wroclaw, Poland. Email: beata.wojtczak@umed.wroc.pl.

\begin{abstract}
Clinical or subclinical primary hyperparathyroidism (PHPT) is one of the most common endocrine disorders in the world. Parathyroidectomy is the treatment of choice in symptomatic patients, stabilizing the progression of complications caused by the destruction activity of parathyroid hormone (PTH). Parathyroid surgical techniques have evolved over the years from bilateral neck exploration, to minimally invasive single parathyroid gland exploration, to minimally invasive video-assisted parathyroidectomy. Localization of the abnormal parathyroid gland before surgery is crucial for the operation to be effective. Increased incidences of reoperations of the parathyroid glands and the rapid development of minimally invasive methods led to the development of new localization techniques. The noninvasive studies include ultrasound (US), computed tomography (CT), magnetic resonance (MR) and ${ }^{99 \mathrm{~m}} \mathrm{Tc}-\mathrm{methoxyisobutylisonitrile}$ (MIBI) scintigraphy combined with single-photon emission CT (SPECT/CT). Among the latest technologies, the four-dimensional (4D)-CT scan, positron emission tomography (PET)/CT and PET/MR are very promising, and are going to have surgical implications in the future.
\end{abstract}

Keywords: Parathyroid imaging; surgical treatment; hyperparathyroidism; parathyroidectomy

Submitted Oct 11, 2019. Accepted for publication Nov 07, 2019.

doi: $10.21037 /$ gs.2019.11.10

View this article at: http://dx.doi.org/10.21037/gs.2019.11.10

\section{Background}

Clinical or subclinical primary hyperparathyroidism (PHPT) is one of the most common endocrine disorders in the world. Since the 1970s, when measuring serum calcium and parathormone became routine, we have observed an increasing number of patients with PHPT (1). In the United States, the prevalence has jumped from 0.08 to about 0.5 per 1,000 , and in Europe the prevalence is about 3 per 1,000 overall, particularly affecting individuals aged $55-75$ years $(2,3)$. PHPT is characterized by excessive secretion of parathyroid hormone (PTH), usually by enlarged parathyroid glands. PHPT is most frequently caused by a single adenoma ( $89 \%$ ); hyperplasia of all the parathyroid glands or double adenomas are less frequently observed ( $6 \%$ and $4 \%$ respectively); in extremely rare cases it is caused by carcinoma (4). Among the many symptoms of PHPT, the most common is recurring nephrolithiasis due to hypercalcemia and low serum phosphate, osteoporosis, fractures, profound weakness and the involvement of other organs, e.g., neuropsychiatric abnormalities, depression, gastrointestinal and cardiovascular symptoms-all of which reduce the quality of life (5). Increasingly, however, especially in developed countries, we observe patients with asymptomatic PHPT or nontypical syndromes (6).

Generally, parathyroidectomy is the treatment of choice in symptomatic patients, stabilizing the progression of complications caused by the destruction activity of 
PTH. However, there are some controversies regarding the indications for parathyroidectomy in asymptomatic patients. Over the past two decades the criteria used to qualify asymptomatic patients for parathyroidectomies have been reevaluated. The latest criteria for surgery of the parathyroid glands include serum calcium more than $1.0 \mathrm{mg} / \mathrm{dL}$ above the reference limit, creatinine clearance $<60 \mathrm{cc} / \mathrm{min}$, markedly reduced bone density at any site (a T-score $<-2,5$ or fragility fracture), and age less than 50 years (7). Some cases with secondary hyperparathyroidism (S-HPT) and tertiary hyperparathyroidism (T-HPT) are indicated for parathyroidectomy as well.

S-HPT is usually caused by chronic renal failure. Chronic renal insufficiency results from phosphorus retention. A strict inverse balance exists between serum levels of phosphorus and calcium, so that phosphorus retention induces hypocalcemia, which stimulates PTH secretion and parathyroid enlargement. Other causes of S-HPT include vitamin D deficiency or intestinal malabsorption. T-HPT refers to the development of autonomous parathyroid hyperfunction, characterized by hypercalcemic hyperparathyroidism in patients with preexisting S-HPT. Bone pain, skeletal deformities (renal osteodystrophy), muscle weakness, growth retardation and extraskeletal calcifications are the main clinical manifestations of this disorder $(5,8)$.

Persistent or recurrent hyperparathyroidism is another indication for parathyroidectomy; it occurs in $5-10 \%$ of patients following primary parathyroidectomy. If the serum calcium level is elevated in the immediate postoperative period, persistent hyperparathyroidism is recognized. It is caused by a failure to localize the abnormal parathyroid gland, inadequate resection of unrecognized multi-gland disease, or the presence of metastatic parathyroid carcinoma. Familial hyperparathyroidism, especially multiple endocrine neoplasia type 1 (the MEN1 syndrome), usually predisposes the patient to persistent hyperparathyroidism. In cases when hyperparathyroidism reappears after a period exceeding 6 months after surgery, recurrent hyperparathyroidism is recognized (9).

Since the first successful parathyroidectomy was performed in 1925 with bilateral neck exploration, it came to be viewed as the treatment of choice in PHPT (10). Parathyroid surgical techniques have evolved over the years from bilateral neck exploration, to minimally invasive single parathyroid gland exploration, to minimally invasive videoassisted parathyroidectomy (11).

Localization of the abnormal parathyroid gland before surgery is crucial for the operation to be effective. The main difficulty in locating the parathyroid glands is their unpredictable location resulting from migration in the embryonic period. In $80 \%$ of patients, the parathyroids are located on the posterior capsule of the thyroid, above and inferior to the crossing of the recurrent laryngeal nerve and inferior thyroid artery (11-13). In $15 \%$ they are located on the posterolateral surface of the superior thyroid gland. In $5 \%$ of cases the parathyroids more posterior in the neck, in retropharyngeal or retroesophageal position. Symmetry is more often observed in the superior parathyroid glands; only $70 \%$ of the inferior glands are symmetric (14). About $0.5-4 \%$ of all parathyroid glands are hidden in the thyroid. Anomalies in parathyroid numbers are observed in 3-6\% of individuals $(5,13,14)$.

Many years ago, when parathyroid surgery was first developed, preoperative diagnoses of the parathyroid glands were not as important as the experience and skill of the surgeon performing the operation, particularly in cases of PHPT. Bilateral exploration of the neck was usually the main way to localize parathyroid pathologies. But increased incidences of reoperations of the parathyroid glands and the rapid development of minimally invasive methods led to the development of new localization techniques (15). Nowadays various noninvasive and invasive imaging studies are available. The noninvasive studies include ultrasound (US), computed tomography (CT), magnetic resonance (MR) and ${ }^{99 \mathrm{~m}} \mathrm{Tc}-\mathrm{methoxyisobutylisonitrile} \mathrm{(MIBI)} \mathrm{scintigraphy}$ combined with single-photon emission CT (SPECT/CT). Invasive studies, usually reserved for reoperative cases, include US or CT-guided fine-needle aspiration (FNA) with concomitant PTH assay, parathyroid angiography and selective venous sampling (SVS) for the PTH gradient (11). Among the latest technologies, the four-dimensional (4D)CT scan, positron emission tomography (PET)/CT and $\mathrm{PET} / \mathrm{MR}$ are very promising, and are going to have surgical implications in the future.

\section{Neck US}

Ultrasonography was introduced in the late $1970 \mathrm{~s}$ particularly for diagnosing PHPT. As a noninvasive, inexpensive method with no radiation or contrast, US is usually still the first method used before surgery to localize parathyroid abnormalities. Generally, a normal parathyroid gland cannot infallibly be visualized with US, but enlarged hypercellular adenomas have a distinct appearance that allows them to be detected. Typical US features of 
parathyroid adenomas are homogeneous hypoechoicity, ovoid shape, hypervascularity in power Doppler imaging and, often, an extrathyroidal feeding vessel entering the parathyroid gland at one of the poles $(16,17)$. US is most useful in identifying parathyroid adenomas that are close to the thyroid gland, but less useful in cases of abnormal tissue localized ectopically in the neck, behind the trachea/ esophagus, or in the mediastinum. The sensitivity and positive predictive value (PPV) of US for detecting enlarged parathyroid glands ranges from $51 \%$ to $96 \%$, and the specificity is $71 \%(18-20)$. US is quick, noninvasive, safe and less expensive than other imaging methods. However, several factors decrease the sensitivity and PPV of US, for example concomitant thyroid nodules, multiple parathyroid adenomas or parathyroid hyperplasia $(21,22)$. It is possible to use ultrasonography intraoperatively to more precisely locate parathyroid adenomas or for US-guided fineneedle biopsy to measure the PTH level, which can help to differentiate an intrathyroid parathyroid adenoma from thyroid nodules (22). The disadvantages of this method include its low value in cases of non-anatomical localizations (substernal, retrotracheal or intramediastinal) of parathyroid glands and its inability to distinguishing parathyroid gland from lymph nodes $(15,23)$. Another limitation of this technique is operator-dependent. According to many authors, USs performed by parathyroid surgeons have been shown to have a higher sensitivity than radiologistperformed parathyroid ultrasonography (24-26).

\section{CT, 4D-CT}

Contrast-enhanced CT is a valuable radiological imaging tool for localizing parathyroid adenomas. The main advantage of this method is that scanning from the base of the skull to the mediastinum allows the detection of ectopic adenomas. The most difficult to find are small parathyroid adenomas, because lymph nodes or tortuous vessels may be mistaken for them. The reported sensitivity of CT ranges from $46 \%$ to $87 \%(19,27,28)$.

4D-CT is a novel method of multiphase CT imaging, characterized by rapid, easily reproducible high-resolution images of anatomic details, useful for surgical planning. The name comes from 3-dimensional (3D)-CT with the addition of a fourth dimension based on changes in contrast perfusion in the tissues over time, which helps identify adenomas more clearly than normal 3D-CT. Parathyroid adenomas have rapid uptake and early washout of intravenous contrast compared to the thyroid. Typically, the 4D-CT protocol for parathyroid adenoma localization includes a pre-contrast scan, an arterial phase scan (25-30 seconds after the contrast bolus), a venous phase scan (approximately 30 seconds after the arterial phase scan) and a delayed phase scan (about 30 seconds after the venous phase scan). The reported sensitivity of 4D-CT scans is as high as $88 \%$ (29). The main challenge in the interpretation of 4D-CT images is differentiating parathyroid adenomas from normal lymph nodes, thyroid nodules and vascular structures. Vu et al. showed that parathyroid adenomas had lower baseline density than thyroid tissue, a greater increase in density from baseline to the arterial phase than the thyroid gland, and much faster contrast washout in the venous and delayed phases than the thyroid (30). Careful observation during the early arterial phase allows vascular structures to be distinguished from parathyroid adenomas. The lymph nodes, in turn, show no enhancement in the arterial phase (31). Eichhorn-Wharry et al. showed that 4DCT is particularly successful in cases of mild hypercalcemia, elevated PTH and smaller adenomas (32).

\section{Magnetic resonance}

Another type of radiological imaging used to locate parathyroid adenomas is MR, which is especially useful for patients who require reoperation because of persistent or recurrent hyperparathyroidism. In MR imaging a typical parathyroid adenoma is an enhancing lesion with isointensity on T1-weighted images and increased signal intensity on T2-weighted images. However, on both T1and $\mathrm{T} 2$-weighted images, parathyroid adenomas may appear isointense or enhanced (33). In the past, 1.5 Tesla and nondedicated protocols were followed, and the sensitivity of MR was reported as $43-71 \%$, comparable to CT (34). As in CT scans, in MR imaging enlarged lymph nodes, thyroid nodules or tortuous vessels can cause false positives (35). The development of 3 Tesla MR and dedicated protocols for the neck improved the sensitivity of parathyroid adenoma detection. Argiro et al. compared the effectiveness of ${ }^{99 \mathrm{~m}} \mathrm{Tc}$ MIBI and 3 Tesla MR imaging in parathyroid adenoma localization; they reported the sensitivity of 3 Tesla MR as $97.7 \%$, the specificity as $97.5 \%$, with PPV $93.7 \%$ and 


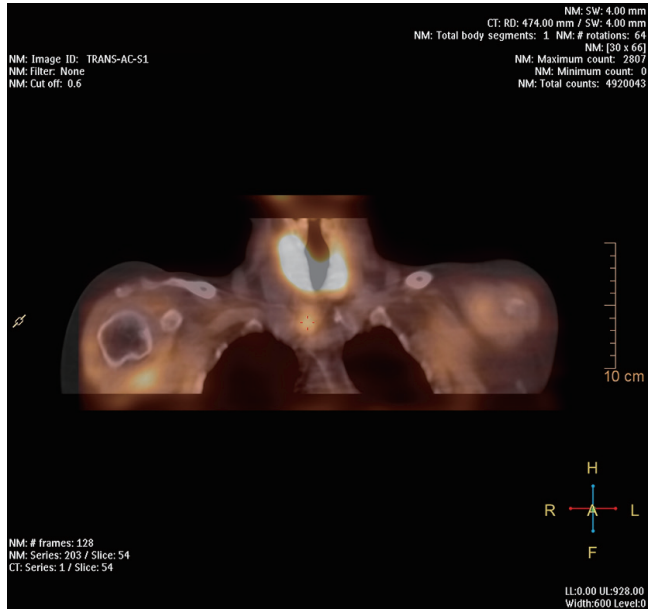

Figure 1 Parathyroid adenoma under right lobe of thyroid- ${ }^{99 \mathrm{~m}} \mathrm{Tc}-$ MIBI SPECT/CT. MIBI, methoxyisobutylisonitrile; SPECT, single-photon emission computed tomography; CT, computed tomography.

negative predictive value (NPV) $99.2 \%$ (36).

\section{Nuclear medicine imaging \\ ${ }^{99 m} T c-M I B I$ scintigraphy}

The most common nuclear medicine imaging technique for preoperative localization of parathyroid adenomas is ${ }^{99 \mathrm{~m}} \mathrm{Tc}-$ MIBI scintigraphy. This method based on the preferential uptake of sestamibi in mitochondria-rich parathyroid adenoma cells and no uptake in normal functioning parathyroid glands. Two different acquisition methods have been used: subtraction and dual-phase images. In the subtraction method, a radiotracer specific to thyroid, such as ${ }^{99 \mathrm{~m}} \mathrm{Tc}$-pertechnetate or ${ }^{123}$ iodium, is subtracted from a scan with ${ }^{99 \mathrm{~m}} \mathrm{Tc}-\mathrm{MIBI}$ to enhance parathyroid adenomas. Dual-phase imaging is based on early and delayed images following intravenous administration of ${ }^{99 \mathrm{~m}} \mathrm{Tc}-\mathrm{MIBI}$ (10-15 minutes vs. 2-3 hours). Parathyroid adenomas exhibit increased uptake and persistence of radiotracer activity, which is washed out of normal thyroid tissue (37). Unfortunately, in up to $30 \%$ of patients this characteristic washout is not seen (38). Three imaging methods have been used in parathyroid scintigraphy: 2-dimensional planar imaging, 3D SPECT and SPECT/CT combined with lowdose CT (SPECT/CT). The hybrid SPECT/CT method has been shown to be superior to normal SPECT, dualphase planar and subtraction scintigraphy because of the combined functional and anatomical information.

At present, ${ }^{99 \mathrm{~m}} \mathrm{Tc}-\mathrm{MIBI}$ SPECT/CT is the most frequently used nuclear imaging method (Figure 1). However, it gives false negatives in as many as 30\% of cases (39). The sensitivity of ${ }^{99 \mathrm{~m}} \mathrm{Tc}-\mathrm{MIBI}$ SPECT/CT scintigraphy ranges from $61 \%$ to $79 \%$, and the specificity is $71 \%$. It is more reliable in identifying larger parathyroid adenomas $(>1.8 \mathrm{~cm})(40)$; the most frequent cause of falsenegative scintigraphy results are smaller parathyroid adenomas (41). The most difficult is to localize are small parathyroid adenomas $(<1.4 \mathrm{~mL})$, ectopic adenomas, adenomas in patients with coexistent multinodular goiter and in patients who have undergone primary neck surgery. Functional imaging with ${ }^{99 \mathrm{~m}}$ Tc-MIBI scintigraphy may give false positives, because some hyperplastic thyroid lesions have extended retention of ${ }^{99 \mathrm{~m}} \mathrm{Tc}-\mathrm{MIBI}$. The most important factors influencing the PPV of scintigraphy are the presence of concomitant thyroid nodules and the size of the parathyroid adenoma. Each method has its strong and weak points, but the sensitivity of combined ${ }^{9 \mathrm{~m}} \mathrm{Tc}-\mathrm{MIBI}$ SPECT/CT scintigraphy is as high as $80 \%$ (42).

${ }^{99 \mathrm{~m}} \mathrm{Tc}-\mathrm{MIBI}$ is also the only radiotracer used to localize parathyroid adenomas by intraoperative radioguidance. An intraoperative gamma-probe detector is most successful 2-3 hours after intravenous tracer injection. Norman et al. carried out scintigraphy on the day of surgery, administering $740 \mathrm{MBq}$ of ${ }^{99 \mathrm{~m}} \mathrm{Tc}-\mathrm{MIBI}$ intravenously. Dualphase scintigraphy was performed 20 minutes and 2 hours after the injection; after approximately $2.5-3$ hours, gammaprobe guided surgery began $(43,44)$. Casara et al. performed ${ }^{99 \mathrm{~m}}$ Tc-MIBI scintigraphy a few days before surgery; then low-dose $37 \mathrm{MBq}{ }^{99 m} \mathrm{Tc}-\mathrm{MIBI}$ was ok administered intravenously just before the start of the operation, and intraoperative PTH (IOPTH) monitoring was used to confirm complete removal of the parathyroid adenoma $(45,46)$. Although ${ }^{99 \mathrm{~m}} \mathrm{Tc}$-sestamibi scans are now the most common nuclear medicine imaging technique used for preoperative localization in PHPT, a more sensitivity nuclear medicine imaging technique is needed.

\section{PET/CT and PET/MR}

Hybrid PET and CT scanning is one of the most accurate imaging methods for diagnosis because of the fusion of anatomical data from CT and metabolic information from PET (Figure 2). PET/CT is used for diagnosis in oncology, cardiology and neurology. Replacing CT with MR, hybrid $\mathrm{PET} / \mathrm{MR}$ imaging is usually better for diagnosing or 


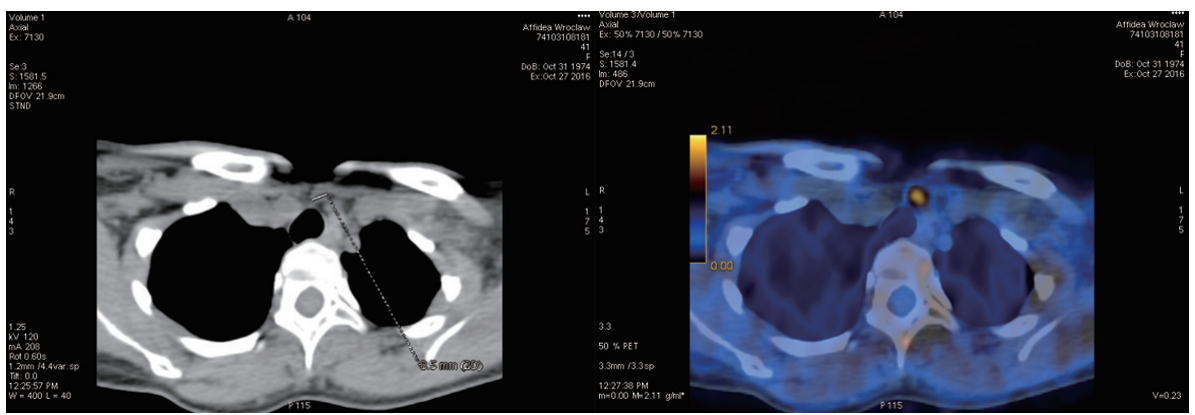

Figure 2 Parathyroid adenoma $-{ }^{18}$ F-fluorocholine PET/CT. PET, positron emission tomography; CT, computed tomography.

assessing diseases that are better seen in MR imagingfor example prostatic cancer. Many different radiotracers are used in PET imaging. To diagnose parathyroid adenomas, the most promising radiotracer appears to be ${ }^{18} \mathrm{~F}$-flurocholine $\left({ }^{18} \mathrm{FCH}\right)$. Choline plays an essential role in the biosynthesis of the phospholipids of cell membranes. So far, ${ }^{18} \mathrm{FCH}$ PET/CT has been used for the staging and restaging of patients with clear cell renal carcinoma and prostate cancer. The currently available data show an increased accumulation of choline in parathyroid adenomas as well as in hyperplastic parathyroid glands. Broos et al. showed that the highest sensitivity of ${ }^{18} \mathrm{FCH} \mathrm{PET/CT}$ was $97 \%$ (47). In a prospective dual-center study with 100 patients with PHPT, Beheshti et al. compared the sensitivity ${ }^{18} \mathrm{FCH}$ PET/CT and ${ }^{99 \mathrm{~m}} \mathrm{Tc}-\mathrm{MIBI}$ scintigraphy. ${ }^{18} \mathrm{FCH}$ PET/ CT imaging was performed 60 minutes after intravenous injection of $3.2 \mathrm{MBq}$ of ${ }^{18} \mathrm{FCH}$ per $\mathrm{kg}$ of body weight. The sensitivity of PET/CT was $93.7 \%$, which was markedly greater than that of ${ }^{99 \mathrm{~m}} \mathrm{Tc}-\mathrm{MIBI}$ SPECT/CT, which showed a sensitivity of $60.8 \%$. The specificity, PPV, NPV and overall accuracy of ${ }^{18} \mathrm{FCH}$ PET/CT in the detection of parathyroid adenomas were $93.7 \%, 96.0 \%, 90.2 \%, 97.4 \%$ and $95.3 \%$ respectively (48). Huber et al. published the results of 26 patients with PHPT who underwent ${ }^{18} \mathrm{FCH}$ PET/CT or PET/MR scanning. Adenomas were detected in 25 patients $(96.2 \%)$, confirmed intraoperatively, and none of the patients needed revision surgery during the follow-up (49). In 2017, Kluijfhout et al. published the results of a prospective pilot study of ${ }^{18} \mathrm{FCH} \mathrm{PET} / \mathrm{MR}$ imaging in ten patients with negative or inconclusive results of US and ${ }^{99 \mathrm{~m}} \mathrm{Tc}-\mathrm{MIBI}$ scintigraphy scans for parathyroid adenomas. After intravenous administration of $3 \mathrm{MBq} /$ $\mathrm{kg}$ of ${ }^{18} \mathrm{FCH}$, PET imaging was performed, as well as T1and T2-weighted MR imaging before and after contrast enhancement. The authors showed $90 \%$ sensitivity and
$100 \%$ PPV for ${ }^{18} \mathrm{FCH}$ PET/MR. The median adenoma SUVmax was 4.9, which was significantly higher than the thyroid SUVmax (2.7) $(\mathrm{P}=0.008)(50)$.

\section{Conclusions}

Parathyroid imaging should be considered only when a diagnosis of hyperparathyroidism and indications for surgery have been established and the patient has agreed to undergo surgery $(11,37)$. Historically, four-gland parathyroid exploration during bilateral neck revision by an experienced surgeon was the standard of care. Nowadays surgeons are focused on smaller incisions and less softtissue dissection, so minimally invasive parathyroidectomy (MIP) is performed in the majority of patients with hyperparathyroidism. MIP has changed surgeons' views on the necessity of preoperative parathyroid diagnoses, since it is possible only with a precise location established preoperatively. Moreover, precise preoperative localization is crucial for patients undergoing reoperation or in cases of persistent hyperparathyroidism $(1,6,11,15,37)$.

There are two different algorithms for localizing parathyroid pathologies in first-time operations and in cases of recurrence or persistent disease (11). In a primary parathyroidectomy accurate localization allows surgeons to perform a minimally invasive procedure. When a biochemical diagnosis of hyperparathyroidism is confirmed in patients with or without symptoms, US and ${ }^{99 \mathrm{~m}} \mathrm{Tc}$-sestamibi scans are usually performed; two positive and concordant examinations allow surgeons to finetune parathyroid surgery. If one of the images is negative, focused exploration with IOPTH measurement or unilateral exploration for two parathyroid glands is recommended. If both the US and the ${ }^{99 \mathrm{~m}} \mathrm{Tc}$-sestamibi are negative, or when they are or discordant, bilateral exploration with IOPTH 
Table 1 The comparison of advantages and disadvantages of different parathyroid imaging techniques

\begin{tabular}{lll}
\hline Examinations & Advantages & Disadvantages \\
\hline First-line & $\begin{array}{l}\text { Noninvasive, inexpensive, no radiation or } \\
\text { contrast, easily accessible } \\
\text { Low-dose radiation }\end{array}$ & Sensitivity, operator-dependent \\
${ }^{99 m}$ TC-MIBI SPECT/CT & Sensitivity, depend of size of parathyroid adenoma \\
Second-line & Higher sensitivity, successful in cases of mild & Higher dose of radiation \\
CT and 4D-CT & hypercalcemia and smaller adenomas & \\
MR & Higher sensitivity, especially useful for patients & Less availability \\
PET/CT and PET/MR & Higher require reoperation & \\
\hline
\end{tabular}

US, ultrasound; MIBI, methoxyisobutylisonitrile; SPECT, single-photon emission computed tomography; CT, computed tomography; PET, positron emission tomography; MR, magnetic resonance.

measurement is the standard protocol (51).

Re-operation of the parathyroid glands is a surgical coup de maitre requiring not only perfect localization, but also supporting documentation such as previous surgical and pathology reports. The localization study includes many tests, ranging from the noninvasive (US, ${ }^{99 \mathrm{~m}} \mathrm{Tc}$ MIBI, CT, MR, PET/CT, PET/MR) to the invasive (SVS and FNA) (11,51). FNA parathyroid biopsy was first described in the 1980s $(52,53)$. The main advantage of FNA biopsy is the high specificity in distinguishing parathyroid and non-parathyroid tissues. This test should not be used before primary operations, as it is an invasive method. Measuring the PTH levels of the aspirate is more sensitive than FNA cytology, which could be mistaken for follicular thyroid tissue (54). The development of the PTH radioimmunoassay led to SVS. The accuracy of this method is $83 \%$, and the sensitivity with intact PTH assay (IPTH) is about $95 \%$ (21). SVS is reserved for parathyroid reoperation in case of negative, equivocal or conflicting noninvasive studies. Technically SVS is a very difficult examination, requiring an experienced interventional radiologist. Fine needle biopsy (FNB) is considered the first invasive procedure to try in cases where US and ${ }^{99 \mathrm{~m}} \mathrm{Tc}-\mathrm{MIBI}$ fail to provide the two concordant tests needed before parathyroid reoperation can commence. SVS should be considered only where CT, 4D-CT, MR, PET/CT or PET/MR are unavailable $(11,37)$. The comparison of advantages and disadvantages of different parathyroids imaging techniques is shown in Table 1.

Finally, the choice of parathyroid imaging method requires knowledge of the radiation dose given. Effective dose of ${ }^{99 \mathrm{~m}} \mathrm{Tc}-\mathrm{MIBI}$ SPECT is about $6 \mathrm{mSv}$ when administered radioactivity is $740 \mathrm{MBq}$. If low dose CT is added to the SPECT effective dose increases to about 8-9 mSv. Absorbed dose for thyroid is about $4 \mathrm{mGy}$. The correct choice of radiation dose is particularly important in patients where parathyroid gland disorders coexist with thyroid disease. The dose of the radiation dose given after 4D-CT could block iodine uptake of the thyroid for many weeks $(14,55)$.

To sum up, there is no perfect imaging method for the parathyroid gland, and selecting the best preoperative imaging to localize parathyroid glands is difficult. The sensitivities of imaging techniques vary significantly from institution to institution, depending on the levels of expertise and variations in the patient populations. Moreover, the results vary between high- and low-volume centers (41). Hyperparathyroidism is seldom a problem in the general population, with a prevalence of about $0.7 \%$. There are usually few centers in any given country where high-volume surgeons perform these operations (42). That is why there is no single superior way to diagnose parathyroid pathology before surgery. High-definition US is still the main examination considered basic in preoperative localization imaging for PHPT. The use of more than one imaging method usually provides more accurate preoperative localization. Among the new techniques, the most promising seem to be 4D-CT and PET/CT or PET/ MR scanning, but neither of these is widely available in in low-volume centers. In the majority of centers, ${ }^{99 \mathrm{~m}} \mathrm{Tc}-$ 
MIBI scans are the most frequently performed, although they have a low rate of sensitivity compared to SPECT/ CT $(11,37,41)$. Possible imaging techniques and their implications for surgical treatment should be considered in the context of overall expenses: a higher initial imaging cost imaging may result in higher rates of intraoperative parathyroid localization, lowering the overall costs of parathyroid treatment. Finally, it should be mentioned that the choice of a given imaging method to much extent is determined by the availability of a given test in a local healthcare environment. Unfortunately, imaging techniques such as CT, 4D-CT, MR, PET/CT or PET/MR are still unavailable in many small centers all over the world.

\section{Acknowledgments}

Acknowledgements for photo PET/CT to Zbigniew Podgajny, PhD, Affidea PET Wrocław.

\section{Footnote}

Conflicts of Interest: The authors have no conflicts of interest to declare.

Ethical Statement: The authors are accountable for all aspects of the work in ensuring that questions related to the accuracy or integrity of any part of the work are appropriately investigated and resolved.

\section{References}

1. Sywak MS, Robinson BG, Clifton-Bligh P, et al. Increase in presentations and procedure rates for hyperparathyroidism in Northern Sydney and New South Wales. Med J Aust 2002;177:246-9.

2. Melton LJ 3rd. The epidemiology of primary hyperparathyroidism in North America. J Bone Miner Res 2002;17 Suppl 2:N12-7.

3. Adami S, Marcocci C, Gatti D. Epidemiology of primary hyperparathyroidism in Europe. J Bone Miner Res 2002;17 Suppl 2:N18-23.

4. Ruda JM, Hollenbeak CS, Stack BC. A systematic review of the diagnosis and treatment of primary hyperparathyroidism from 1995 to 2003. Otolaryngol Head Neck Surg 2005;132:359-72.

5. Mariani G, Gulec SA, Rubello D, et al. Preoperative localization and radioguided parathyroid surgery. J Nucl Med 2003;44:1443-58.
6. Pallan S, Khan A. Primary hyperparathyroidism: update on presentation, diagnosis, and management in primary care. Can Fam Physician 2011;57:184-9.

7. Bilezikian JP, Khan AA, Potts JT Jr. Third International Workshop on the Management of Asymptomatic Primary Hyperthyroidism: 2009 Summary Statement: Guidelines for the Management of Asymptomatic Primary Hyperparathyroidism: Summary Statement from the Thirchap 58d International Workshop. J Clin Endocrinol Metab 2009;94:335-9.

8. Fraser WD. Hyperparathyroidism. Lancet 2009;374:145-8.

9. Metz D, Jensen R, Allen B, et al. Multiple endocrine neoplasia type 1: clinical features and management. In: Bilezikian J, Levine M, Marcus R. editors. The Parathyroids. New York, NY: Raven Press, 1994:591-647.

10. Mandl F. Therapeutic attempt of osteitis fibrosa generalisata by excision of an epithelial-corpuscle tumors. Wien Klin Wochenschr 1925;195:1343-4.

11. Mitmaker EJ, Grogan RH, Duh QY. Guide to preoperative parathyroid localization testing. In: Randolph G. editor. Surgery of the thyroid and parathyroid glands. Philadelphia: Saunders, 2012:539-45.

12. Herrera MF, Gamboa-Dominguez A. Parathyroid embryology, anatomy, and pathology. In: Clark OH, Duh QY. editor. Textbook of endocrine surgery. Philadelphia: WB Saunders, 1997:277-83.

13. Randolph GW, Urken ML. Surgical management of primary hyperparathyroidism. In: Randolph GW. Surgery of the thyroid and parathyroid glands. Philadelphia: Saunders, 2003:507-28.

14. Akerström G, Malmaeus J, Bergström R. Surgical anatomy of human parathyroid glands. Surgery 1984;95:14-21.

15. Duh QY, Sancho JJ, Clark OH. Parathyroid localization. Clinical review. Acta Chir Scand 1987;153:241-54.

16. Shindo ML. Parathyroid ultrasonography. Head \& Neck Ultrasonography. 2nd edition. San Diego, CA: Plural Publishing, 2016:137-54.

17. Johnson NA, Tublin ME, Ogilvie JB. Parathyroid imaging: technique and role in the preoperative evaluation of primary hyperparathyroidism. AJR Am J Roentgenol 2007;188:1706-15.

18. Rodriquez JM, Tezelman S, Siperstein AE, et al. Localization procedures in patients with persistent or recurrent hyperparathyroidism. Arch Surg 1994;129:870-5.

19. Khan AA, Hanley DA, Rizzoli R, et al. Primary hyperparathyroidism: review and recommendations on 
evaluation, diagnosis, and management. A Canadian and international consensus. Osteoporos Int 2017;28:1-19.

20. Powell AC, Alexander HR, Chang R, et al. Reoperation for parathyroid adenoma: a contemporary experience. Surgery 2009;146:1144-55.

21. Mihai R, Simon D, Hellman P. Imaging for primary hyperparathyroidism - an evidence-based analysis. Langenbecks Arch Surg 2009;394:765-84.

22. Barczynski M, Golkowski F, Konturek A, et al. Technetium-99m-sestamibi subtraction scintigraphy vs. ultrasonography combined with a rapid parathyroid hormone assay in parathyroid aspirates in preoperative localization of parathyroid adenomas and in directing surgical approach. Clin Endocrinol (Oxf) 2006;65:106-13.

23. Fine EJ. Parathyroid imaging: its current status and future role. Semin Nucl Med 1987;17:350-9.

24. Whelan PJ, Rotstein LE, Rosen JB, et al. Do we really need another localizing technique for parathyroid glands? Am J Surg 1989;158:382-4.

25. Van Husen R, Kim LT. Accuracy of surgeon-performed ultra- sound in parathyroid localization. World J Surg 2004;28:1122-6.

26. Soon PS, Delbridge LW, Sywak MS, et al. Surgeon performed ultrasound facilitates minimally invasive parathyroidectomy by the focused lateral mini-incision approach. World J Surg 2008;32:766-71.

27. Aydin C, Polat SB, Dellal FD, et al. The diagnostic value of parathyroid hormone washout in primary hyperparathyroidism patients with negative or equivocal 99m'Tc-MIBI results. Diagn Cytopathol 2019;47:94-9.

28. Ahuja AT, Wong KT, Ching AS, et al. Imaging for primary hyperparathyroidism-what beginners should know. Clin Radiol 2004;59:967-76.

29. Rodgers SE, Hunter GJ, Hamberg LM, et al. Improved preoperative planning for directed parathyroidectomy with 4-dimensional computed tomography. Surgery 2006;140:932-40.

30. Vu TH, Guha-Thakurta N, Harrel RK, et al. Imaging characteristics of hyperfunctioning parathyroid adenomas using multiphase multidetector computed tomography: a quantitative and qualitative approach. J Comput Assist Tomogr 2011;35:560-7.

31. Ellika S, Patel S, Aho T. Preoperative localization of parathyroid adenomas using 4-dimensional computed tomography: a pictorial essay. Can Assoc Radiol J 2013;64:258-68.

32. Eichhorn-Wharry LI, Carlin AM, Talpos GB. Mild hypercalcemia: an indication to select 4-dimensional computed tomography scan for preoperative localization of parathyroid adenomas. Am J Surg 2011;201:334-8.

33. Fakhran S, Branstetter BF, Pryma DA. Parathyroid imaging. Neuroimaging Clin N Am 2008;18:537-49.

34. Lopez Hänninen E, Vogl TJ, Steinmüller T, et al. Preoperative contrast-enhanced MRI of the parathyroid glands in hyperparathyroidism. Invest Radiol 2000;35:426-30.

35. Flickinger FW, Sathyanarayana, White JE. MRI in hyperparathyroidism requiring reoperation. Clin Imaging 1991;15:210-2.

36. Argirò R, Diacinti D, Sacconi B, et al. Diagnostic accuracy of 3T magnetic resonance imaging in the preoperative localisation of parathyroid adenomas: comparison with ultrasound and 99mTc-sestamibi scan. Eur Radiol 2018;28:4900-8.

37. Zafereo M, Yu J, Angelos P, et al. American Head and Neck Society Endocrine Surgery Section update on parathyroid imaging for surgical candidates with primary hyperparathyroidism. Head Neck 2019;41:2398-409.

38. Quak E, Ciappuccini R, Nganoa CA, et al. The performance of dual-phase $99 \mathrm{~m}$ Tc-sestamibi parathyroid SPECT/CT in primary hyperparathyroidism. Eur J Nucl Med Mol Imaging 2014;41:S569.

39. Ciappuccini R, Morera J, Pascal P, et al. Dual-phase 99m Tc sestamibi scintigraphy with neck and thorax SPECT/CT in primary hyperparathyroidism: a singleinstitution Experoence. Clin Nucl Med 2012;37:223-8.

40. Kunstman JW, Kirsch JD, Mahajan A, et al. Clinical review: Parathyroid localization and implications for clinical management. J Clin Endocrinol Metab 2013;98:902-12.

41. Wong KK, Fig LM, Gross MD, et al. Parathyroid adenoma localization with 99mTc-sestamibi SPECT/CT: a meta-analysis. Nucl Med Commun 2015;36:363-75.

42. Lenschow C, Gassmann P, Wenning C, et al. Preoperative (1)(1)C-methionine PET/CT enables focused parathyroidectomy in MIBI-SPECT negative parathyroid adenoma. World J Surg 2015;39:1750-7.

43. Norman JG. Minimally invasive radioguided parathyroidectomy: an endocrine surgeon's perspective. J Nucl Med 1998;39:15N, 24N.

44. Norman J, Denham D. Minimally invasive radioguided parathyroidectomy in the reoperative neck. Surgery 1998;124:1088-92; discussion 1092-3.

45. Casara D, Rubello D, Piotto A, et al. 99mTc-MIBI radio-guided minimally invasive parathyroid surgery planned on the basis of a preoperative combined $99 \mathrm{mc}-$ 
pertechnetate/99m Tc-MIBI and ultrasound imaging protocol. Eur J Nucl Med 2000;27:1300-4.

46. Casara D, Rubello D, Cauzzo C, et al. 99m Tc-MIBI radioguided minimally invasive parathyroidectomy: experience with patients with normal thyroids and nodular goiters. Thyroid 2002;12:53-61.

47. Broos WAM, van der Zant FM, Knol RJJ, et al. Choline PET/CT in parathyroid imaging: a systematic review. Nucl Med Commun 2019;40:96-105.

48. Beheshti M, Hehenwarter L, Paymani Z, et al. $18 \mathrm{~F}$-Fluorocholine PET/CT in the assessment of primary hyperparathyroidism compared with $99 \mathrm{~m}$ Tc-MIBI or 99m Tc-tetrofosmin SPECT/CT: a prospective dualcentre study in 100 patients. Eur J Nucl Med Mol Imaging 2018;45:1762-71.

49. Huber GF, Hüllner M, Schmid C, et al. Benefit of 18 F-fluorocholine PET imaging in parathyroid surgery. Eur Radiol 2018;28:2700-7.

50. Kluijfhout WP, Pasternak JD, Gosnell JE, et al. $18 \mathrm{~F}$ Fluorocholine PET/MR imaging in patients with primary hyperparathyroidism and inconclusive conventional imaging: a prospective pilot study. Radiology
2017;284:460-7.

51. Haciyanli M, Lal G, Morita E, et al. Accuracy of preoperative localization studies and intraoperative parathyroid hormone assay in patients with primary hyperparathyroidism and double adenoma. J Am Coll Surg 2003;197:739-46.

52. Doppman JL, Krudy AG, Marx SJ, et al. Aspiration of enlarged parathyroid glands for parathyroid hormone assay. Radiology 1983;148:31-5.

53. Gooding GA, Clark OH, Stark DD, et al.: Parathyroid aspiration biopsy under ultrasound guidance in the postoperative hyperparathyroid patient. Radiology 1985;155:193-6.

54. Abraham D, Sharma PK, Bentz J, et al. Utility of ultrasound-guided fine-needle aspiration of parathyroid adenomas for localization before minimally invasive parathyroidectomy. Endocr Pract 2007;13:333-7.

55. Moosvi SR, Smith S, Hathorn J, et al. Evaluation of the radiation dose exposure and associated cancer risks in patients having preoperative parathyroid localization. Ann R Coll Surg Engl 2017;99:363-8.
Cite this article as: Wojtczak B, Syrycka J, Kaliszewski K, Rudnicki J, Bolanowski M, Barczyński M. Surgical implications of recent modalities for parathyroid imaging. Gland Surg 2020;9(Suppl 2):S86-S94. doi: 10.21037/gs.2019.11.10 